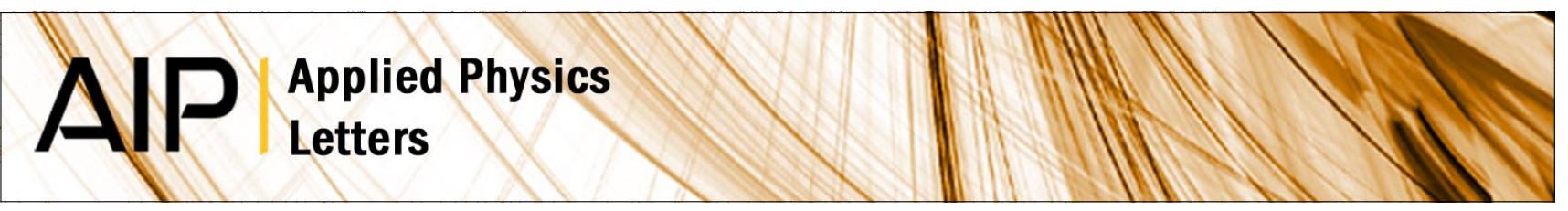

Stability, resolution, and ultra-low wear amplitude modulation atomic force microscopy of DNA: Small amplitude small set-point imaging

Sergio Santos, Victor Barcons, Hugo K. Christenson, Daniel J. Billingsley, William A. Bonass et al.

Citation: Appl. Phys. Lett. 103, 063702 (2013); doi: 10.1063/1.4817906

View online: http://dx.doi.org/10.1063/1.4817906

View Table of Contents: http://apl.aip.org/resource/1/APPLAB/v103/i6

Published by the AIP Publishing LLC.

Additional information on Appl. Phys. Lett.

Journal Homepage: http://apl.aip.org/

Journal Information: http://apl.aip.org/about/about_the_journal

Top downloads: http://apl.aip.org/features/most_downloaded

Information for Authors: http://apl.aip.org/authors

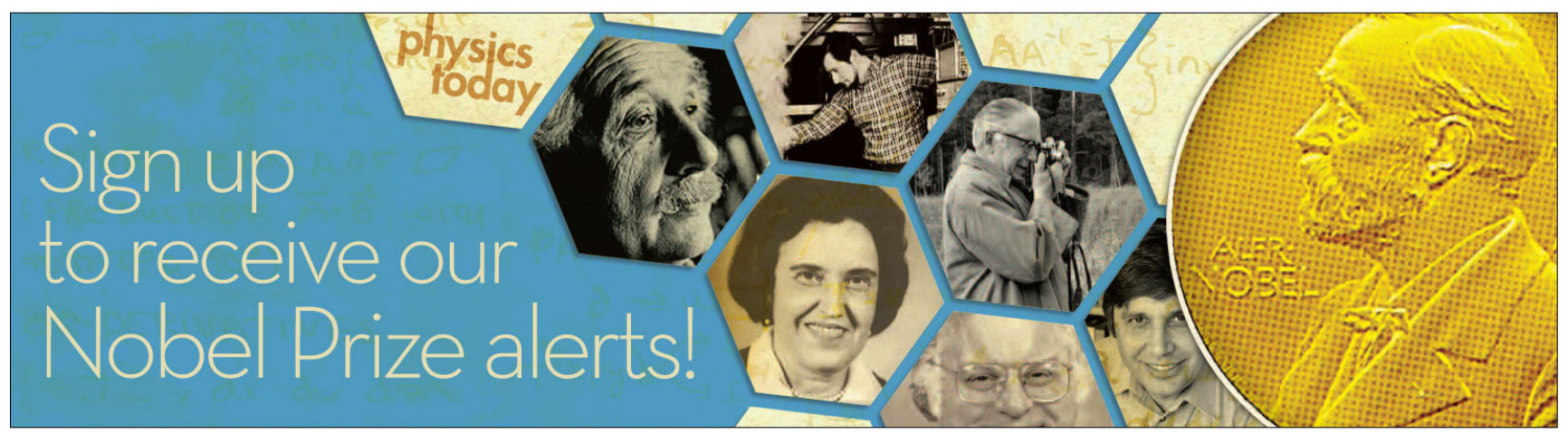




\title{
Stability, resolution, and ultra-low wear amplitude modulation atomic force microscopy of DNA: Small amplitude small set-point imaging
}

\author{
Sergio Santos, ${ }^{1,2,3}$ Victor Barcons, ${ }^{3}$ Hugo K. Christenson, ${ }^{1}$ Daniel J. Billingsley, ${ }^{1,4}$ \\ William A. Bonass, ${ }^{4}$ Josep Font, ${ }^{3}$ and Neil H. Thomson ${ }^{1,4}$ \\ ${ }^{1}$ School of Physics and Astronomy, University of Leeds, Leeds LS2 9JT, United Kingdom \\ ${ }^{2}$ Laboratory for Energy and Nanosciences, Masdar Institute of Science and Technology, Abu Dhabi, \\ United Arab Emirates \\ ${ }^{3}$ Departament de Disseny i Programació de Sistemes Electrònics, UPC-Universitat Politècnica \\ de Catalunya Av. Bases, 61,08242 Manresa, Spain \\ ${ }^{4}$ Department of Oral Biology, Leeds Dental Institute, University of Leeds, Leeds LS2 9LU, \\ United Kingdom
}

(Received 21 April 2013; accepted 19 July 2013; published online 6 August 2013)

\begin{abstract}
A way to operate fundamental mode amplitude modulation atomic force microscopy is introduced which optimizes stability and resolution for a given tip size and shows negligible tip wear over extended time periods $(\sim 24 \mathrm{~h})$. In small amplitude small set-point (SASS) imaging, the cantilever oscillates with sub-nanometer amplitudes in the proximity of the sample, without the requirement of using large drive forces, as the dynamics smoothly lead the tip to the surface through the water layer. SASS is demonstrated on single molecules of double-stranded DNA in ambient conditions where sharp silicon tips $(\mathrm{R} \sim 2-5 \mathrm{~nm})$ can resolve the right-handed double helix. (C) 2013 AIP Publishing LLC. [http://dx.doi.org/10.1063/1.4817906]
\end{abstract}

Amplitude modulation atomic force microscopy (AM AFM) is one of the most broadly employed imaging modes of the AFM family, particularly for air and liquid environments, especially on samples with significant topographical variations ${ }^{1-6}$ since the AM AFM architecture and electronics are relatively simple and robust. ${ }^{1,3,7}$ Despite its rapid success on experimental grounds, however, it has taken much effort to understand cantilever dynamics in fundamental frequency AM AFM. Bi-stability and the related issues involved with the interaction via a non-monotonic, non-linear tip-sample potential, ${ }^{8,9}$ the snap into contact problem ${ }^{3}$ and the possible perturbations related to capillary interactions ${ }^{10,11}$ have made achieving stability and resolution in ambient conditions particularly challenging ${ }^{12}$ as compared to ultra-high vacuum where even bond order discrimination is now possible. ${ }^{13}$

In ambient AM AFM, bi-stability is usually prevalent for larger excitation amplitudes, once the critical amplitude to reach the repulsive force regime has been surpassed. ${ }^{11,14}$ Intermittent contact (IC) between the tip and the sample occurs at these larger amplitudes and often risks tip or sample damage and/or protracted wearing of the tip. ${ }^{15}$ To circumvent these issues, one can significantly lower the excitation amplitude to enable non-contact (NC) imaging conditions. ${ }^{16,17}$ In NC mode, the tip is at much lower risk of damage since only attractive forces operate, and the bi-stable region is not accessed. However, image resolution is generally compromised in $\mathrm{NC}$ mode because the tip is on average further from the sample, increasing the effective interaction area as a consequence of the long range van der Waals forces. ${ }^{3,12,18}$ In terms of resolution, the advantages of removing the large contact areas related to long range forces have been demonstrated both in ultra-high vacuum, ${ }^{19}$ by placing atoms that interact covalently rather than ionically, and in liquid environments, ${ }^{20,21}$ since London dispersion forces are negligible in water as compared to air.
More recently, different schemes have been implemented in multi-frequency (MF) AFM that can provide a variety of imaging channels and can probe material properties of the sample. ${ }^{22-24} \mathrm{MF}$ AFM approaches often require specialized architectures and hardware to detect higher harmonics or to excite higher modes. Harmonic excitation however is strongly coupled to non-linearities, and these provide detailed information about the tip-sample forces. ${ }^{25}$ Still, the excitation of higher harmonics requires tip-sample interactions that might be beyond what soft biological samples might withstand. ${ }^{26}$ From this point of view, understanding and optimizing fundamental mode AM AFM imaging is important in hydrated and liquid environments. ${ }^{26,27}$ In this work, we use cantilevers with a relatively high $\mathrm{Q}$ factor $(\sim 500)$ and intermediate stiffness $(\mathrm{k} \sim 40 \mathrm{~N} / \mathrm{m})$ where excitation of higher frequencies is suppressed and undetectable, using small free amplitudes ( $\sim 1-5 \mathrm{~nm})$.

Here, a path in ambient AM AFM is found that smoothly brings the tip through the water layer at very close tip-surface proximity where images are acquired with subnanometer oscillation amplitude. Typically, ${ }^{11,28}$ in order to enable the tip to interact via the short-range repulsive forces from which high resolution originates, ${ }^{18,19,25}$ large amplitudes (energies) were used to break through the water layer and overcome the prevalent capillary interactions. We report, however, that provided the tip is sharp enough $(\mathrm{R}<5 \mathrm{~nm})$, the tip can reach the surface at once and smoothly, i.e., avoiding bi-stability, with free amplitudes $\left(\mathrm{A}_{0}<5 \mathrm{~nm}\right)$ an order of magnitude smaller than those in standard repulsive mode $\left(\mathrm{A}_{0} \sim 20-50 \mathrm{~nm}\right) .{ }^{11,29-31}$ In this way, stable imaging in the repulsive regime with small oscillation and free amplitudes can be achieved. Small amplitude small set-point (SASS) operates close to the zero (net) sample force. This implies that the average force per cycle is zero or close to zero and, since the oscillation amplitudes are of the order of 
$1 \AA$ and the free amplitudes minimized, peak repulsive forces are also minimized. ${ }^{26}$ The energy dissipated per cycle in SASS $(\sim 1 \mathrm{eV})$ is also an order of magnitude smaller than that of standard $\left(\sim 10-10^{2} \mathrm{eV}\right)$ repulsive imaging. That is, the imaging conditions in SASS are optimal for high resolution imaging of soft matter since conservative and dissipative forces in SASS originate from the more localized repulsive interactions, ${ }^{18}$ while peak forces and energy dissipation are minimized.

Consider a standard experimental amplitude curve (black line) obtained as a function of cantilever separation $\mathrm{z}_{\mathrm{c}}$ with a standard AC160TS (OLYMPUS) cantilever on a mica sample (Fig. 1(a)). Note that while the free or unperturbed amplitude $\mathrm{A}_{0}$ is slightly less than $5 \mathrm{~nm}$, a region of negative slope can be observed when $\mathrm{z}_{\mathrm{c}}<\mathrm{A}_{0}$ (at $\sim \mathrm{Z}_{\mathrm{c}}=2 \mathrm{~nm}$ in this case). For simplicity $z_{c}$ has been arbitrarily set to $0 \mathrm{~nm}$ for the smallest value of A. This region of negative slope in A smoothly connects the two regions of positive slope in A where AM AFM can be stably operated. The region of negative slope simply brings the tip approximately $2 \mathrm{~nm}$ closer to the surface, relative to the first region of positive slope in A or the standard attractive regime of operation, ${ }^{31}$ for values of oscillation amplitude as small as $\mathrm{A}<1 \mathrm{~nm}$. This statement can be confirmed experimentally by monitoring the corresponding minimum distance of approach per cycle $d_{\text {min }}$. Neglecting higher harmonics, $d_{\min }=z_{c}-A$ (grey line). Note that by monitoring $d_{\min }$ it is observed that the negative slope in A serves the purpose of smoothly decreasing $d_{\min }$ in that region (Fig. 1(a)). That is, the negative region of slope in A smoothly drives the tip very close to the surface, past the long range forces, to where the more localized repulsive and dissipative forces are found. ${ }^{18}$ It can also be readily observed in Fig. 1(a) that, after the negative region in slope in A, $d_{\text {min }}$ slightly and also smoothly increases by fractions of nm with decreasing $A$. That is, the absolute minimum in $d_{\text {min }}$ coincides with local maximum in A. The slight increase in $d_{\text {min }}$ past the local minima is responsible for minimizing the tip-sample deformation, peak forces, and energy past the region of negative slope in A. Practically, this is achieved in SASS by decreasing the oscillation amplitude below $1 \mathrm{~nm}$ past the region of negative slope, or local maxima in A, while keeping $\mathrm{A}_{0}$ constant as is common in AM AFM. Experientially, the physics just described can be demonstrated by employing the Sader-Katan formalism ${ }^{32,33}$ (1) to reconstruct the conservative part of the force $\mathrm{F}_{\mathrm{ts}}{ }^{*}$ and the standard energy dissipation expression, ${ }^{34}$ (2) to reconstruct the energy dissipation per cycle $\mathrm{E}_{\mathrm{dis}}{ }^{*}$. Asterisks imply normalizing with the use of the absolute in minima in $\mathrm{F}_{\mathrm{ts}}$ - or adhesion force $\mathrm{F}_{\mathrm{AD}}$ - and maxima in $\mathrm{E}_{\mathrm{dis}}-$ or $\mathrm{E}_{\mathrm{dis}(\mathrm{M})^{-}}$, respectively. For example in Fig. $1, \mathrm{~F}_{\mathrm{AD}} \approx-1.5 \mathrm{nN}$ and $\mathrm{E}_{\mathrm{dis}(\mathrm{M})} \approx 9 \mathrm{eV}$. Furthermore, for simplicity, $\mathrm{d}_{\min }$ is identified with $\mathrm{d}$ throughout and $\Omega$ is the normalized frequency shift. ${ }^{33}$

$$
\begin{gathered}
F_{t s}^{*}(d)=\frac{2 k}{\left|F_{A D}\right|} \int_{u=d}^{u=\infty}\left[\left(1+\frac{A}{8 \sqrt{\pi(u-d)}}\right) \Omega(u)\right. \\
\left.-\frac{A^{3 / 2}}{\sqrt{2(u-d)}} \frac{d \Omega(u)}{d u}\right] d u, \\
E_{d i s}^{*}=\frac{\pi k A_{0} A}{E_{d i s}(M) Q}\left[\sin (\Phi)-\frac{A}{A_{0}}\right] .
\end{gathered}
$$

The above expressions have been solved numerically with MATLAB. ${ }^{35}$ The force reconstruction in Fig. 1(b) (dashed blue lines) can be used to describe the interesting physical phenomena related to the region of negative slope in $\mathrm{A}$ and what occurs past this region. Note that the distance $d$ has been arbitrarily set to $0 \mathrm{~nm}$ when $\mathrm{F}_{\mathrm{ts}}=\mathrm{F}_{\mathrm{AD}}$ as discussed below. As stated, past the local maxima in A (Fig. 1(a)) the minimum

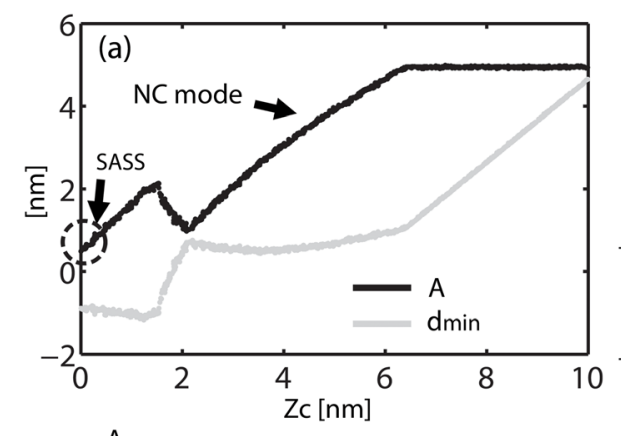

A

(c) NC mode

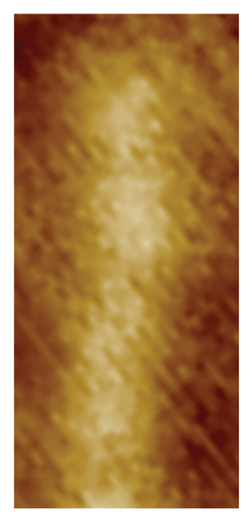

(d) SASS mode

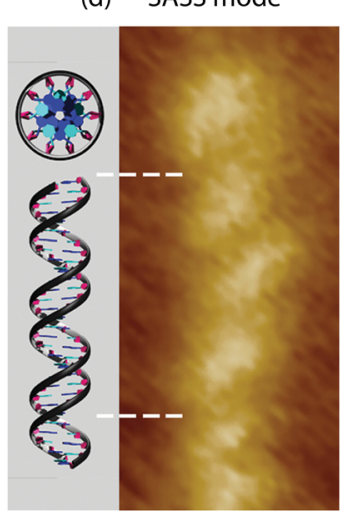

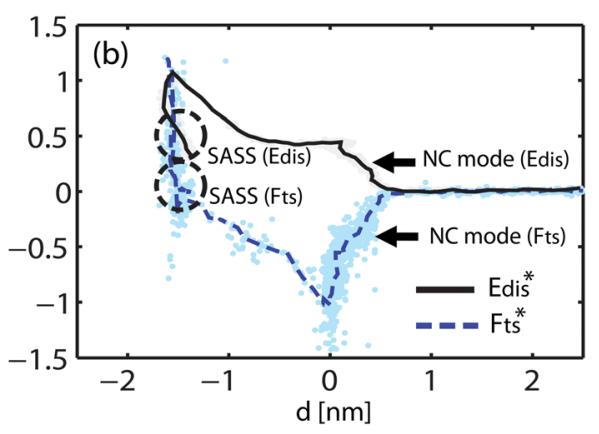

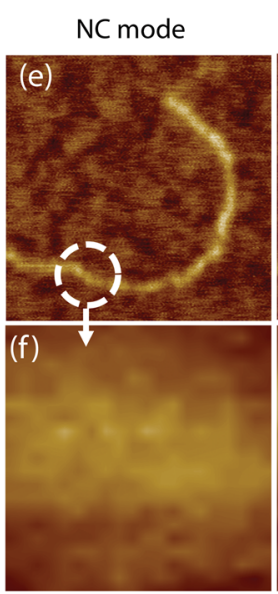

SASS mode

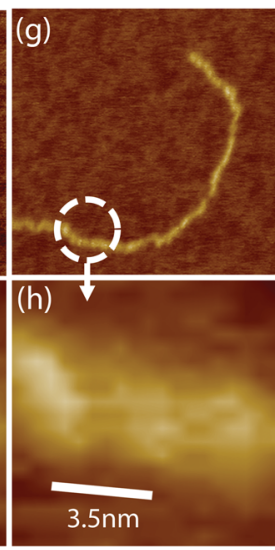

FIG. 1. Experimental (a) amplitude A (back lines) and minimum distance of approach $d_{\min }$ (grey lines) and (b) normalized tip-sample force $\mathrm{F}_{\mathrm{ts}}{ }^{*}$ (dashed blue lines) and energy dissipation $\mathrm{E}_{\mathrm{dis}}{ }^{*}$ (black lines) as a function of cantilever separation $\mathrm{z}_{\mathrm{c}}$ and distance $\mathrm{d}$, respectively. (c)-(h) Experimental examples of images of dsDNA on mica obtained in the NC and SASS modes, respectively. Higher resolution is obtained in the SASS mode provided the tip is sharp enough. Here $\mathrm{R}<5 \mathrm{~nm}$. 
distance of approach d increases by a few $\AA$ with decreasing amplitude A. That is, the repulsive peak forces ${ }^{26}$ and sample deformation decrease $(\sim \AA)$ in SASS as observed in Fig. 1(b). Note that for the smaller values of A, the peak forces lie around the 0 net force region, or below the 0 net region, ${ }^{36}$ i.e., SASS mode for $\mathrm{F}_{\mathrm{ts}}{ }^{*}$ (circle) (Fig. 1(b)). For the same values of $\mathrm{A}$, the energy dissipated $\mathrm{E}_{\mathrm{dis}}{ }^{*}$ (black lines) also decreases for two reasons: (1) because of the increase in $d$ and (2) because of the decrease in tip velocity with decreasing A, i.e., SASS mode for $\mathrm{E}_{\mathrm{dis}}{ }^{*}$ (circle). The net effect is the reduction of peak forces, sample deformation and energy dissipation in the SASS mode with decreasing A. The meaning of the value $d=0 \mathrm{~nm}$ in Fig. 1(b) is discussed next.

In Fig. 1(b) $d=0$ has been set when minima, i.e., $\mathrm{F}_{\mathrm{AD}}$, in force occurs. Also note that Fig. 1(b) indicates that SASS might be operating with the tip inside the water layer (Figs. 1S-3S). ${ }^{36}$ This can be deduced from the fact that there are two distinct negative slopes in $\mathrm{F}_{\mathrm{ts}}{ }^{*}$ for $\mathrm{d}<0$. The region of larger slope can be identified with the true surface. The region of negative slope in $\mathrm{F}_{\mathrm{ts}}{ }^{*}$ where $-1.5<\mathrm{d}<0 \mathrm{~nm}$ (approx.) could then be identified with the region where water layers from the tip and the sample overlap. ${ }^{11,37}$ Very recent work suggests that this interpretation and phenomena is also observed in frequency modulation AFM. ${ }^{38}$ Imaging inside these nanometric water layers could thus provide a protective effect to the tip and the surface and may be a consequence of lubricity in the nanoscale contact. The tip size in this work was independently determined using a recent in situ tip sizing method which uses the onset of bi-stability. ${ }^{14}$ The low-wear involved with imaging in the SASS mode, and possibly related to imaging inside the water layers, is discussed later in Fig. 2, experimentally. Suffice it to say however that such low wear imaging mode might have implications in other fields such as data storage. ${ }^{39}$

In the context of soft matter imaging, the water layers on the hydrophilic mica surface also ensure that the DNA retains sufficient hydration to remain in the B-form helix. Arguably, imaging single DNA molecules is also a bench mark for high resolution imaging of soft matter with the AFM. ${ }^{40}$ Figs. 1(c) and 1(d) show a comparison of the resolution for a region of an individual double-stranded DNA molecule on a mica surface in NC mode (Fig. 1(c)) and in SASS mode (Fig. 1(d)). In Fig. 1(c) (NC mode) the outline of the molecule is observed but sub-molecular features are not readily resolved. With the SASS mode however ${ }^{36}$ (Fig. 1(d)) the right-handed helix of the DNA is resolved and compared to a molecular model of B-form DNA at the same magnification. The parameters in the figure were $\mathrm{A}_{0} \approx 3 \mathrm{~nm}$ and $\mathrm{A} \approx 2.5 \mathrm{~nm}$ ( $\mathrm{NC}$ mode) and $\mathrm{A} \approx 0.1 \mathrm{~nm}$ (SASS mode). Another example and a similar comparison is provided in Figs. 1(e)-1(h). While SASS can resolve the DNA helical periodicity and correct chirality to a high degree of precision, it is as yet not possible to routinely resolve the double helix along its entire length. This is principally for two reasons, (1) current sample preparation procedures for ambient tend to leave salt deposits over parts of the molecules and (2) that commercial silicon tips might, even if slightly, i.e., $\sim 1 \mathrm{~nm}$, vary in sharpness. Moreover, ingenuity by the user is still required in SASS since even small, i.e., pm, variations in A and $\mathrm{A}_{0}$ will result in similar variations in $\mathrm{d}_{\min }$ that control resolution for a given tip radius.

SASS also conveys another important advantage over typical AM modes: it is very insensitive to changes in the cantilever resonance due to thermal or other effects. This is demonstrated in Fig. 2 which compares NC mode with SASS mode. The NC mode (Fig. 2(a)) is in the small amplitude regime $\left(\mathrm{A}_{0}=2 \mathrm{~nm}\right)$, and after $30 \mathrm{~min}$ or less the tip is no longer tracking the surface. In SASS however, the tip can scan across the surface for $\approx 24 \mathrm{~h}$ with no loss of resolution or loss of surface tracking. Monitoring the tip in situ ${ }^{14}$ while performing the experiments further showed that there was negligible tip damage after acquiring the images in Fig. 2. As a final note, the physics behind the requirement of sharp tips for SASS imaging, i.e., with sub-nanometer oscillation amplitudes and in the proximity of the surface, might relate to the increase in tip-sample forces with increasing tip radius $\mathrm{R}$ for a given tip-sample distance. That is, at a given distance

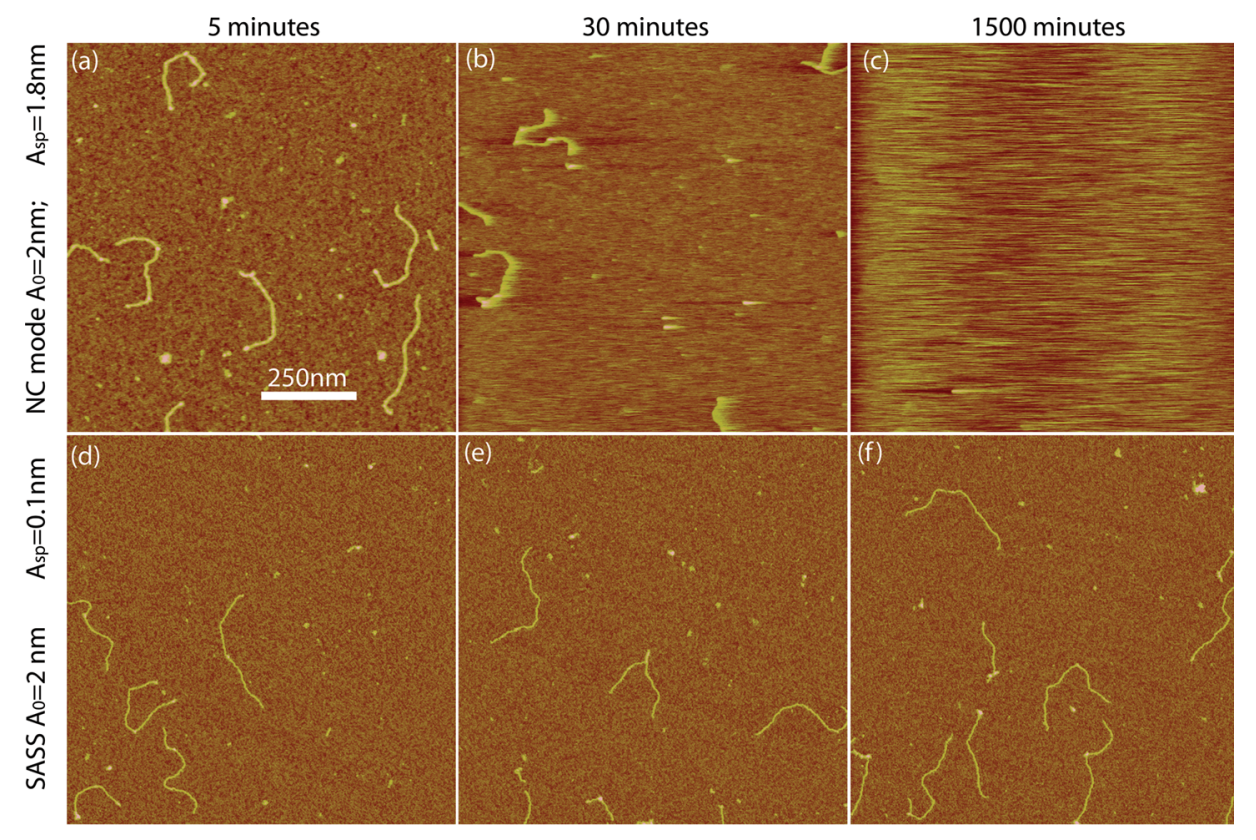

FIG. 2. Experimental example of images of dsDNA on mica obtained in the (a)-(c) NC and (d)-(f) SASS modes, respectively. Only the SASS mode allows stable imaging for $25 \mathrm{~h}$ without signs of tip wear or problems related to thermal drift of the cantilever resonance curve. The total distance that has been travelled by the tip in (f) without tip wear is $36 \mathrm{~cm}$. 
the tip-sample forces escalate with $\mathrm{R}$. Thus, if sufficient energy is not provided by the driving force (free amplitude in AM AFM) and sufficient energy is not stored in the cantilever (related to oscillation amplitude in AM AFM), the dynamics of the cantilever cannot be controlled by the feedback system and SASS fails. We have in fact observed (data not shown) that the sharper tips allow employing SASS with the smallest values of oscillation and free amplitude.

In summary, for SASS the tip oscillates in the region close to the surface where resolution is maximized, while tip-sample deformation, peak forces and energy dissipation is minimized by approximately an order of magnitude relative to standard repulsive mode. Recall that the area of interaction due to van der Waals forces rapidly increases with separation. ${ }^{18,19}$ A region of relatively large tip-sample distances is where NC mode operates. If the distance is decreased to the point where deformation occurs, initially the short range forces decrease the interaction area but further decreasing the distance results in deformations for which the effective area of interaction increases again. This is where standard repulsive or IC mode imaging operates. There is thus a location that lies in between these two where a high resolution instrument should operate. This is in fact the location which we show SASS operates, although the actual resolution obtained depends on the tip size, the local sample conditions and slight variations in $\mathrm{d}_{\min }$ controlled by $\mathrm{A}$ and $\mathrm{A}_{0}$. In addition to the small force and energy interactions, in ambient conditions the SASS region might further coincide with the overlapping of the nanometric tip-sample water layers apparently largely increasing its low tip-wear capabilities. Low tip-wear has implications in all fields of metrology by scanning probe microscopy including datastorage, as well as high resolution imaging of soft matter.

${ }^{1}$ R. Garcia and R. Perez, Surf. Sci. Rep. 47, 197-301 (2002).

${ }^{2}$ F. Moreno-Herrero, J. Colchero, and A. Baro, Ultramicroscopy 96, 167-174 (2003).

${ }^{3}$ F. J. Giessibl, Rev. Mod. Phys. 75, 949-983 (2003).

${ }^{4}$ Q. Zhong, D. Inniss, K. Kjoller, and V. B. Elings, Surf. Sci. Lett. 290,

L688-L692 (1993).

${ }^{5}$ C. F. Quate, Surf. Sci. 299-300, 980-995 (1994).

${ }^{6} \mathrm{C}$. Bustamante and D. Keller, Phys. Today 48(6), 32 (1995).

${ }^{7}$ R. Garcia, Amplitude Modulation Atomic Force Microscopy (Wiley-VCH, Weinheim, 2010).

${ }^{8}$ R. W. Stark, Mater. Today 13(9), 24-32 (2010).

${ }^{9}$ A. S. Paulo and R. Garcia, Phys. Rev. B 66(4), 041406 (2002).
${ }^{10}$ A. 1. Weisenhorn, P. K. Hansma, T. R. Albrecht, and C. F. Quate, Appl. Phys. Lett. 54, 2651-2653 (1989).

${ }^{11}$ S. Santos, A. Verdaguer, T. Souier, N. H. Thomson, and M. Chiesa, Nanotechnology 22(46), 465705 (2011).

${ }^{12}$ Y. Gan, Surf. Sci. Rep. 64(3), 99-121 (2009).

${ }^{13}$ L. Gross, F. Mohn, N. Moll, B. Schuler, A. Criado, D. P. Guitián, A. Gourdon, and G. Meyer, Science 337, 1326-1329 (2012).

${ }^{14}$ S. Santos, L. Guang, T. Souier, K. R. Gadelrab, M. Chiesa, and N. H. Thomson, Rev. Sci. Instrum. 83, 043707 (2012).

${ }^{15}$ S. Santos and N. H. Thomson, Appl. Phys. Lett. 98, 013101 (2011).

${ }^{16}$ A. San Paulo and R. Garcia, Biophys. J. 78, 1599-1605 (2000).

${ }^{17}$ R. Garcia and A. San Paulo, Phys. Rev. B 61, R13381-R13384 (2000).

${ }^{18}$ S. Santos, V. Barcons, A. Verdaguer, J. Font, N. H. Thomson, and M. Chiesa, Nanotechnology 22, 345401 (2011).

${ }^{19}$ L. Gross, F. Mohn, N. Moll, P. Liljeroth, and G. Meyer, Science 325, 1110-1114 (2009).

${ }^{20}$ K. Voïtchovsky, J. J. Kuna, S. A. Contera, E. Tosatti, and F. Stellacci, Nat. Nanotechnol. 5, 401-405 (2010).

${ }^{21}$ T. Fukuma, K. Kobayashi, K. Matsushige, and H. Yamada, Appl. Phys. Lett. 86, 193108 (2005).

${ }^{22}$ D. Martinez-Martin, E. T. Herruzo, C. Dietz, J. Gomez-Herrero, and R. Garcia, Phys. Rev. Lett. 106, 198101 (2011).

${ }^{23}$ R. Garcia and E. T. Herruzo, Nat. Nanotechnol. 7, 217-226 (2012).

${ }^{24}$ A. Raman, S. Trigueros, A. Cartagena, A. P. Z. Stevenson, M. Susilo, E. Nauman, and S. A. Contera, Nat. Nanotechnol. 6, 809-814 (2011).

${ }^{25}$ F. J. Giessibl, Mater. Today 8(5), 32-41 (2005).

${ }^{26}$ H. V. Guzman, A. P. Perrino, and R. Garcia, ACS Nano 7(4), 3198-3204 (2013).

${ }^{27}$ E. Wutscher and F. J. Giessibl, Rev. Sci. Instrum. 82, 093703 (2011).

${ }^{28}$ S. Santos, A. Verdaguer, and M. Chiesa, J. Chem. Phys. 137, 044201 (2012).

${ }^{29}$ S. Santos, V. Barcons, J. Font, and N. H. Thomson, J. Phys. D 43, 275401 (2010).

${ }^{30}$ S. Santos, V. Barcons, J. Font, and N. H. Thomson, Nanotechnology 21(22), 225710 (2010).

${ }^{31}$ R. Garcia and A. S. Paulo, Phys. Rev. B 60(7), 4961-4967 (1999).

${ }^{32}$ J. E. Sader and S. P. Jarvis, Appl. Phys. Lett. 84, 1801-1803 (2004).

${ }^{33}$ A. J. Katan, M. H. van Es, and T. H. Oosterkamp, Nanotechnology 20, 165703 (2009).

${ }^{34}$ J. P. Cleveland, B. Anczykowski, A. E. Schmid, and V. B. Elings, Appl. Phys. Lett. 72(20), 2613-2615 (1998).

${ }^{35}$ T. M. MATLAB R2010b and SIMULINK, Inc., Natick, Massachusetts, USA.

${ }^{36}$ See supplementary material at http://dx.doi.org/10.1063/1.4817906 for examples of $\mathrm{NC}$ and SASS imaging at different values of relative humididy and the corresponding force reconstruction.

${ }^{37}$ V. Barcons, A. Verdaguer, J. Font, M. Chiesa, and S. Santos, J. Phys. Chem. C 116(14), 7757-7766 (2012).

${ }^{38}$ D. S. Wastl, A. J. Weymouth, and F. J. Giessibl, Phys. Rev. B 87, 245415 (2013).

${ }^{39}$ E. Gnecco, Nature (London) 461, 178-179 (2009).

${ }^{40}$ A. Cerreta, D. Vobornik, and G. Dietler, Eur. Polym. J. 49(8), 1916-1922 (2013). 